\title{
PADRÕES DE DEFICIÊNCIA HÍDRICA PARA A CULTURA DE MILHO (SAFRA NORMAL E SAFRINHA) NO ESTADO DE GOIÁS E SUAS CONSEQÜENCIAS PARA O MELHORAMENTO GENÉTICO
}

\author{
Water stress pattern for corn (first and second crop) in the Goiás State and their \\ consequences for the breeding program
}

\author{
Alexandre Bryan Heinemann¹, Camilo de Lelis Teixeira de Andrade², Reinaldo Lúcio Gomide, \\ André de O. Amorim ${ }^{4}$, Rosidalva Lopes da $\mathrm{Paz}^{5}$
}

\begin{abstract}
RESUMO
Deficiência hídrica é considerada a maior restrição na produção e estabilidade da produtividade de culturas em muitas regiões do mundo. No Estado de Goiás, região na qual predomina a produção em sequeiro, para a cultura do milho (Zea mays L.) implantada na safra normal e na safrinha, é comum sofrer períodos de estresse por deficiência hídrica intermitente ou terminal, que reduzem o rendimento de grãos. No processo de desenvolvimento de novos híbridos e variedades cultivadas, genótipos são selecionados em função de sua adaptabilidade em um determinado ambiente alvo. Assim, programas de melhoramento vegetal, com o objetivo de desenvolver híbridos e variedades cultivadas mais adaptados a um determinado ambiente, requerem informações sob a probabilidade de ocorrência dos diferentes tipos de deficiência hídrica, como também, suas características, intensidade e tempo, em função da fase fenológica da cultura. Um modelo de simulação de culturas foi utilizado para determinar os padrões de deficiência hídrica no estado de Goiás, considerando 12 locais e 6 diferentes datas de semeadura para a cultura do milho semeada na safra normal e na safrinha. Para a cultura do milho semeado na safra normal, a perda na produtividade decorrente do estresse por deficiência hídrica foi menor que $50 \%$, sendo que os tipos de deficiência hídrica que provocam um maior impacto na produtividade iniciam-se no começo do período reprodutivo. Para o milho semeado na safrinha, a perda na produtividade é superior a 50\%, sendo mais comum o estresse terminal, que tem sua maior intensidade no enchimento de grãos.
\end{abstract}

Termos para indexação: Modelos de crescimento, Zea mays, cerrado, veranico, estresse hídrico.

\section{ABSTRACT}

Water stress is a major constraint to crop production and yield stability in many regions of the world. The cultivation of corn (Zea mays L.) in the Brazilian State of Goiás, is frequent affected by periods of water stress resulting in yield reduction. During the process of developing new hybrids and cultivated varieties, new genotypes are selected based on their adaptability for a certain environment. In this context, plant breeding programs demand for information regarding as a function of the crop phenological phases. A crop simulation model was used to determine patterns of water stress for 12 locations of the State of Goiás and 6 different sowing for both, first and second corn harvest periods. For the first harvest period, yield loss due to water stress was lower than 50\%, with higher effects on yield in the beginning of the reproductive period. For corn as a second crop, yield loss is higher than $50 \%$ with frequent occurrence of terminal stress, which presented higher intensity during the stage of grain filling.

Index terms: Growth model, Zea mays, Brazilian savanna, dry spells, water stress.

(Recebido em 29 de novembro de 2007 e aprovado em 21 de outubro de 2008)

\section{INTRODUÇÃO}

A cultura de milho é um importante componente da cadeia produtiva de suínos e aves instalada no estado de Goiás. No ano de 2007, Goiás produziu cerca de 2,85 milhões de toneladas de milho, sendo $78 \%$ na safra normal e $22 \%$ na safrinha (IBGE, 2007). Em ambos os períodos, a produção majoritária é de sequeiro e é comum a cultura sofrer períodos de estresse por deficiência hídrica intermitente ou no final do ciclo, contribuindo para a redução do rendimento de grãos. $\mathrm{O}$ efeito negativo da deficiência hídrica pode ser

\footnotetext{
Engenheiro Agrônomo, Doutor - Embrapa Arroz e Feijão CNPAF - Rod. GO-462, Km 12, Zona Rural - 75375-000 - Santo Antônio de Goiás, GO alexbh@cnpaf.embrapa.br

Engenheiro Agrícola, Ph.D - Núcleo de Solo, Água e Sustentabilidade Ambiental/NSAM - Embrapa Milho e Sorgo - Rod. MG 424, km 45, Zona Rural Cx.P. 151 - 35701-970 - Sete Lagoas, MG - camilo@cnpms.embrapa.br

${ }^{3}$ Engenheiro Agrônomo, Engenharia Agrícola, Engenharia de Irrigação, Ph.D - Embrapa Milho e Sorgo -Embrapa Milho e Sorgo, Embrapa/CNPMS - Rod. MG 424 Km 45, s/n, Zona Rural - Cx. P. 151 - 35701-970 - Sete Lagoas, MG - gomide@cnpms.embrapa.br

${ }^{4}$ Geógrafo, Mestrando em Engenharia Agrícola - Sistema de Meteorologia e Hidrologia do Estado de Goiás/SIMEHGO - Secretaria de Estado de Ciência e Tecnologia/SECTEC - Rua 82 s/no , Palácio Pedro L. Teixeira, 2o Andar Setor Sul - 74088-900 - Goiânia, GO - amorim_go@yahoo.com..br

${ }^{5}$ Bacharel em Física, Mestranda em Engenharia do Meio Ambiente - Sistema de Meteorologia e Hidrologia do Estado de Goiás/SIMEHGO - Secretaria de Estado de Ciências e Tecnologia/SECTEC - Rua 82 s/nº Palácio Pedro L. Teixeira, º Andar Setor Sul - 74088-900 - Goiânia, GO - dalvapaz@yahoo.com.br
} 
agravado pelo desenvolvimento limitado do sistema radicular da cultura, decorrente da toxidez de alumínio observada nas camadas subsuperficiais do perfil dos solos típicos de cerrado.

No processo de desenvolvimento de novos híbridos e variedades cultivadas, genótipos-alvos são selecionados em função da sua adaptabilidade a um determinado ambiente, mediante ensaios de campo de avaliação e seleção de genótipos denominados VCU (valor de cultivo e uso), conduzidos em diferentes locais. Os diversos ensaios de VCU procuram captar as interações genótipo versus ambiente (GxA), incluindo as variações espaciais e temporais. Esse conjunto de ambientes, incluindo suas variações espaciais e temporais, no qual um determinada variedade cultivada desenvolvida pelo programa de melhoramento necessita se adaptar, é definido como "target population of environments" (TPE) ou população de ambientes alvos (Cooper et al., 1997). Considerando que uma TPE não é homogênea em razão, principalmente, dos elementos climáticos, especialmente a precipitação pluvial, é esperado encontrar interações entre genótipos e ambiente, ocasionando variações na performance dos genótipos nos diferentes locais de ensaios. Um programa de melhoramento com o objetivo de desenvolver híbridos e variedades cultivadas adaptados a uma determinada região requer informações sobre a probabilidade de ocorrência dos diferentes tipos de deficiência hídrica, bem como suas características como intensidade e duração e época de ocorrência em função da fase fenológica da cultura. Trata-se, portanto, da caracterização ambiental, levando-se em consideração, não somente os aspectos climáticos básicos como precipitação pluvial, mas também, os efeitos da interação GxA. O conhecimento das características de uma TPE pode facilitar a escolha de sítios representativos para a realização dos ensaios VCUs, assim como auxiliar a interpretação dos resultados de ensaios em função dos diferentes padrões de deficiência hídrica ocorrida. Uma melhor caracterização da TPE, definindo-se os padrões de estresses por deficiência hídrica para um determinado ambiente é, portanto, uma importante ferramenta para apoiar decisões no planejamento dos programas de melhoramento vegetal. Nos últimos anos, a caracterização indireta, que considera a avaliação do desempenho das culturas em função das variações ambientais (Cooper \& Fox, 1996), vem-se tornando comum com a utilização de modelos de simulação de crescimento de culturas. Chapman et al. (2000b) utilizaram o modelo APSIM, baseado em informações locais de solo e dados climáticos, para determinar a tipologia da deficiência hídrica para o sorgo em Queensland, Austrália.
Neste estudo, objetivou-se caracterizar as variações geográficas e temporais dos padrões de deficiência hídrica para a cultura de milho, semeado na safra normal e na safrinha no Estado de Goiás, e os seus respectivos impactos no programa de melhoramento genético de milho.

\section{MATERIAL E MÉTODOS}

\section{Caracterização da Área de Estudo}

Neste trabalho, a área de estudo considerada foi de doze municípios do Estado de Goiás. O clima é considerado quente, semi-úmido com uma precipitação pluvial anual de $1600 \mathrm{~mm}$ concentrada entre sete e oito meses (Nimer, 1989). Os municípios considerados (Aragarças, Ceres, Sto. Antônio de Goiás, Goiânia, Ipora, Itaberaí, Itumbiara, Planaltina, Porangatu, Quirinópolis, Vianópolis e Vicentinópolis) foram selecionados para estabelecer a base de dados meteorológicos e, consequentemente, a TPE. As informações da localização geográfica, altitude e número de anos da série histórica dos dados meteorológicos utilizados estão ilustradas na Tabela 1. As variáveis meteorológicas diárias: temperatura máxima e mínima do ar, precipitação pluvial, umidade relativa máxima e mínima do ar, velocidade do vento e radiação global foram obtidas da rede de estações do SECTEC/SIMEHGO (Secretaria do Estado de Ciência e Tecnologia - Goiás). Na área de estudo, o solo mais frequente, representando $46 \%$ da área total, é o Latossolo (Embrapa, 1999), caracterizado por elevada permeabilidade e aeração, o que lhe confere propriedades físicas adequadas para o cultivo do milho. Sua capacidade de água disponível por unidade de profundidade efetiva do sistema radicular e de água é de aproximadamente $100 \mathrm{~mm} \mathrm{~m}^{-1}$ (Schaffert et al., 2000; Balbino et al., 2001). Nesse tipo de solo, há limitação para o desenvolvimento do sistema radicular de algumas culturas em razão do elevado nível de Alumínio (Al) tóxico nas camadas subsuperficiais do perfil do solo. Assim, dois cenários para solos foram estabelecidos: a) CAD $100 \mathrm{~mm}$ (solo com capacidade de água disponível de $100 \mathrm{~mm}$ para uma profundidade efetiva de $1 \mathrm{~m}$ ) e b) CAD 50 $\mathrm{mm}$ (solo com capacidade de água disponível de $50 \mathrm{~mm}$ para uma profundidade efetiva de $0,5 \mathrm{~m}$ ), com a intenção de reproduzir o efeito da toxidez de $\mathrm{Al}$.

\section{Modelo de Simulação de Crescimento de Cultura}

O modelo de crescimento CEREAL 06, implementado na plataforma de modelos ECOTROP (Sultan et al., 2005; Kouressy et al., 2008), foi parametrizado por meio de dados experimentais obtidos da variedade de ciclo médio-curto BRS 3003. Esse é um modelo determinístico, com passos diários de cálculo, desenvolvido com base no modelo SARRA, utilizado para análise de risco climático das culturas de sorgo e milheto na África Oriental (Affholder, 1997). 
Tabela 1 - Municípios e suas respectivas; coordenadas geográficas (latitude e longitude, em graus), altitude (m) e número de anos utilizados no estudo (n).

\begin{tabular}{|c|c|c|c|c|}
\hline Local & Latitude* & Longitude* & Altitude (m) & $\mathrm{n}$ \\
\hline Aragarças & $-16,00$ & $-52,00$ & 310 & 6 \\
\hline Sto A. de Goiás & $-16,50$ & $-49,30$ & 741 & 22 \\
\hline Ceres & $-15,33$ & $-49,60$ & 739 & 6 \\
\hline Goiânia & $-16,73$ & $-49,25$ & 749 & 6 \\
\hline Iporá & $-16,41$ & $-51,11$ & 688 & 6 \\
\hline Itaberai & $-16,01$ & $-49,78$ & 1001 & 6 \\
\hline Itumbiara & $-18,40$ & $-49,18$ & 449 & 6 \\
\hline Planaltina & $-16,08$ & $-47,70$ & 1007 & 6 \\
\hline Porangatu & $-13,30$ & $-41,11$ & 391 & 6 \\
\hline Quirinópolis & $-18,43$ & $-50,40$ & 633 & 6 \\
\hline Vianópolis & $-16,80$ & $-48,48$ & 1110 & 6 \\
\hline Vicentinópolis & $-17,70$ & $-49,78$ & 648 & 6 \\
\hline
\end{tabular}

*Os valores negativos representam a convenção de latitude sul e longitude oeste.

\section{Dinâmica da água no solo}

O modelo utilizado simula o balanço diário da água no solo. O solo é dividido em duas camadas, sendo a primeira camada, geralmente de $10 \mathrm{~cm}$, utilizada para o cálculo da evaporação, e a segunda (profundidade definida pelo usuário) representa a camada cujo limite inferior é a profundidade máxima que o sistema radicular pode alcançar (Baron et al., 1996). A quantidade de água disponível no solo é baseada na diferença entre a "capacidade de campo" e "ponto de murcha permanente" e provém de dados obtidos no laboratório, sendo considerada neste estudo $100 \mathrm{~mm} \mathrm{~m}^{-1}$. A profundidade efetiva do sistema radicular depende das características genótipicas e da fase fenológica da cultura e é limitada de acordo com a cultura, solo e profundidade de umedecimento do perfil do solo. A extração da água do solo consiste basicamente em dois componentes: evaporação pela superfície do solo e extração pelas raízes, via transpiração.

\section{Intensidade do estresse por deficiência hídrica na planta}

A deficiência hídrica da planta é avaliada por meio da fração da água disponível do solo na zona das raízes, removida via transpiração. A variável FTSW, que é a relação entre a lâmina de água transpirada e a lâmina de água disponível na zona efetiva radicular, normalizada para a escala 0 a 1, é calculada diariamente (Sinclair \& Ludlow, 1986). Essa variável atua como um fator de redução no cálculo da transpiração e assimilação de carbono, utilizando o fator de depleção $p$ (Allen et al., 1998). A intensidade do estresse por deficiência hídrica da planta é representada pela variável $C S T R$ (transpiração real/transpiração potencial, variando de 0 a 1 ).

\section{Características das Simulações}

As simulações foram efetuadas utilizando-se a mesma variedade para os períodos de semeadura (safra normal e safrinha) do milho no estado de Goiás. Na safra normal, as datas de semeadura foram consideradas de 15 de outubro a 31 de dezembro, com intervalos de 15 dias e, na safrinha, de 20 de janeiro a 1 de março, com intervalos de 10 dias. Em razão do fato de as datas de semeadura terem sido fixadas a priori no modelo, independente da ocorrência de chuva, uma restrição foi imposta, permitindo que a emergência ocorresse somente se a umidade média na primeira camada do solo fosse maior ou igual a $70 \%$ da "capacidade de campo", nos dez dias subsequentes à semeadura. O início das simulações se deu em julho, para permitir um estabelecimento mais realístico do perfil de água do solo, baseado nas ocorrências de precipitação pluvial antes das datas de semeadura.

\section{Tipologia dos padrões de deficiência hídrica}

Para o estabelecimento dos padrões de deficiência hídrica para a cultura do milho na safra normal e safrinha foi elaborada uma matriz constituída de local, data de semeadura e ano (representando as linhas) e a média dos valores do CSTR (intensidade do estresse por deficiência hídrica na planta), definido como a razão entre a transpiração real e transpiração potencial, para cada 
período de 100 GD (graus dia, com temperatura base de $8^{\circ} \mathrm{C}$ ) do desenvolvimento da cultura (representando as colunas). A essa matriz aplicou-se o método de classificação denominado "cluster". Esse método é hierárquico e de aglomeração, baseado na distância euclidiana como medida de dissimilaridade e soma dos quadrados como critério de fusão (Ward, 1963). Para cada TPE, safra normal e safrinha, as tipologias das deficiências hídricas foram classificadas em três principais grupos, baseados na similaridade do CSTR em função do desenvolvimento fenológico simulado da cultura. Para evitar tendências nas análises dos padrões de deficiência hídrica, considerou-se somente para análise o período do ciclo da cultura entre 400 GD (metade do período vegetativo) e o final do enchimento dos grãos. Classificação similar foi utilizada por Chapman et al. (2000a).

As variáveis de saída do modelo foram: CSTR (transpiração real/transpiração potencial), produtividade potencial simulada (PP - sem deficiência hídrica), produtividade real simulada (PR). A partir das variáveis PP e PR, calculou-se o impacto da deficiência hídrica na produtividade, dado pela seguinte equação (1):

$$
I R S H=100\left(1-\frac{P R}{P P}\right)
$$

em que, IRSH se refere ao impacto da deficiência hídrica na produtividade, em $\%, P R$ a produtividade real simulada, em $\mathrm{kg} \mathrm{ha}^{-1}$, e $P P$ a produtividade potencial simulada, em $\mathrm{kg}$ ha $^{-1}$.

O programa estatístico utilizado neste estudo foi o R v.2.4.0 (R Development Core Team, 2005), utilizando-se o pacote "Cluster".

\section{RESULTADOS E DISCUSSÃO}

\section{Caracterização dos padrões de deficiência hídrica - milho safra normal}

Para o milho cultivado na safra normal foram determinados três diferentes padrões de deficiência hídrica em função da análise de cluster (Figura 1a). Esses três padrões foram denominados L (sem deficiência hídrica), M (deficiência hídrica mediana no período reprodutivo) e SR (deficiência hídrica severa no período reprodutivo). O padrão de deficiência hídrica SR foi o mais severo, ocasionando uma perda na produtividade de 81 e $89 \%$, para os cenários de solo CAD $100 \mathrm{~mm}$ e $50 \mathrm{~mm}$, respectivamente (Tabela 2). Esse padrão inicia-se no começo do período reprodutivo (500 GD) e tem a maior intensidade no final dessa fase (900 GD), ou seja, a uma semana antes da data de florescimento. Entretanto, esse padrão apresentou a menor frequência para ambos os solos (Tabela 2). O padrão de deficiência hídrica $\mathrm{M}$ ocasionou uma perda na produtividade de 24 e $29 \%$, para os solos com CAD $100 \mathrm{~mm}$ e $50 \mathrm{~mm}$, respectivamente (Tabela 2). Esse padrão, também, inicia-se no período reprodutivo (600 GD) e se estende até o enchimento dos grãos. Para o solo CAD $50 \mathrm{~mm}$, esse padrão é o mais frequente $(71 \%)$. O padrão de deficiência hídrica $L$ apresentou o menor impacto na produtividade, 1 e $2 \%$ para os solos CAD $100 \mathrm{~mm}$ e 50 $\mathrm{mm}$, respectivamente. Esse padrão é o mais frequente para o cenário CAD $100 \mathrm{~mm}$, representando $62 \%$ dos casos de ocorrência.

As datas de semeadura que apresentaram a maior probabilidade para a ocorrência do padrão de deficiência hídrica SR foram no início e no final do período de semeadura (15/10 e 31/12) para o solo CAD $100 \mathrm{~mm}$. No caso do solo CAD $50 \mathrm{~mm}$, esse tipo de deficiência hídrica (SR) se estende até $1 / 11$. As datas de semeadura que apresentaram o menor índice de deficiência hídrica (L) estão entre 15/11 e 15/12 (Figura 2a) para ambos os solos. Falhas na emergência, decorrente da falta de umidade no solo, foram observadas somente no início do período de semeadura (Figura 2a).

\section{Caracterização dos padrões de deficiência hídrica - milho safrinha}

Para o milho na safrinha, também, foram determinados três diferentes padrões de deficiência hídrica em função da análise de cluster realizada (Figura 1b). Esses três padrões de deficiência hídrica (Figura 1b) foram denominados L (sem deficiência hídrica), T (deficiência hídrica terminal) e RT (deficiência hídrica no período reprodutivo e terminal). $\mathrm{O}$ padrão RT foi o mais severo, ocasionando uma perda na produtividade de 91 e $95 \%$, para os solos CAD $100 \mathrm{~mm}$ e de $50 \mathrm{~mm}$, respectivamente (Tabela 2). Esse estresse tem início no começo do período reprodutivo e possui maior intensidade no enchimento de grãos. Esse padrão também apresentou a segunda maior frequência para o solo CAD 50 mm (34\%). O padrão de deficiência hídrica T ocasionou uma perda na produtividade de 56 e $68 \%$, para os solos com CAD $100 \mathrm{~mm}$ e $50 \mathrm{~mm}$, respectivamente. Esse padrão tem início no período reprodutivo, sendo que, a sua intensidade aumenta no enchimento de grãos. Para ambos os solos, esse padrão de deficiência hídrica é o mais frequente (Tabela 2). O padrão de deficiência hídrica L apresentou o menor impacto na produtividade, 11 e $31 \%$, para os solos CAD 100 $\mathrm{mm}$ e CAD $50 \mathrm{~mm}$, respectivamente. Esse padrão apresenta uma ligeira deficiência hídrica durante o enchimento de grãos, sendo mais intenso no solo CAD $50 \mathrm{~mm}$. 

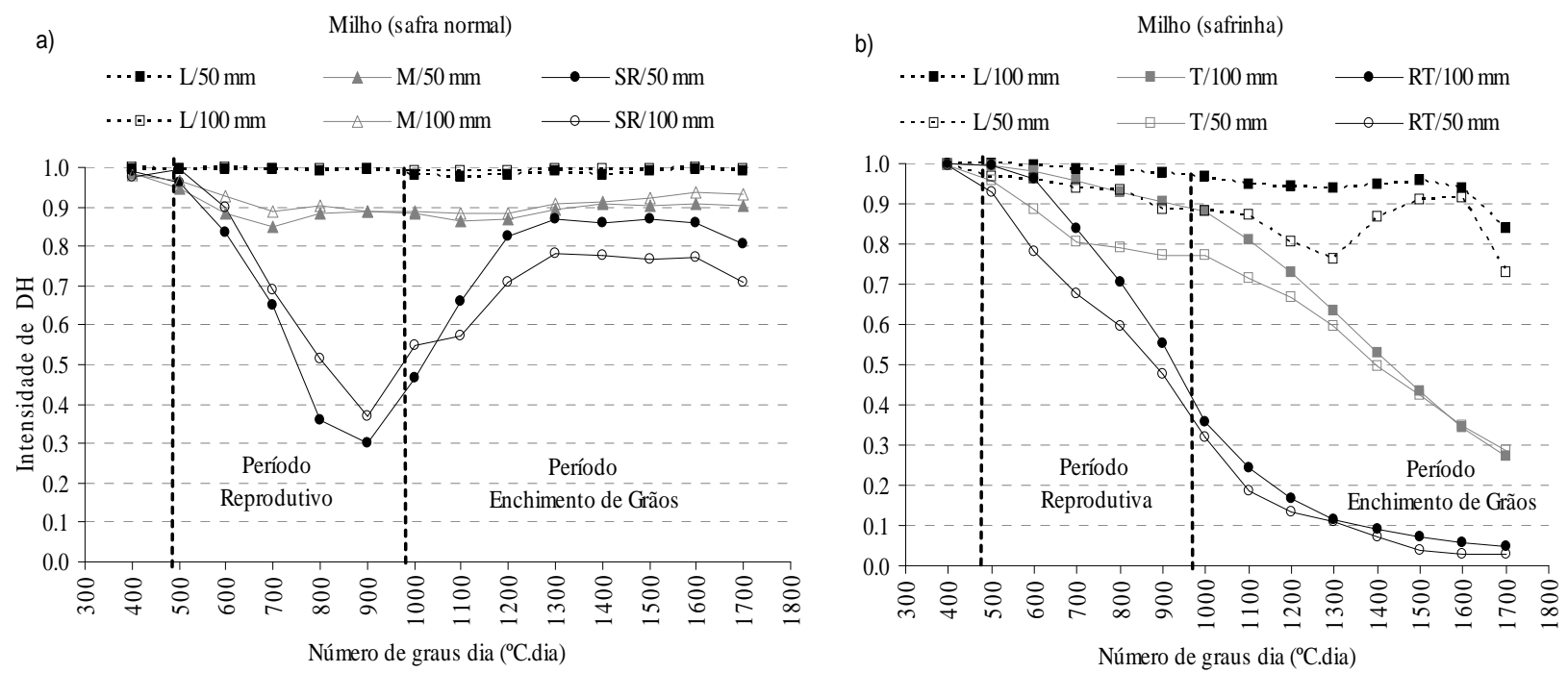

Figura 1 - Padrões de deficiência hídrica (DH) e suas intensidades em função das fases de desenvolvimento da cultura de milho safra normal (a) e safrinha (b), sendo (L) sem deficiência hídrica, (M) deficiência hídrica mediana, (SR) deficiência hídrica severa na fase reprodutiva, (T) deficiência hídrica terminal e (RT) deficiência hídrica na fase reprodutiva e terminal, para os cenários de solos com CAD $100 \mathrm{~mm}$ e $50 \mathrm{~mm}$.

Tabela 2 - Produtividade potencial (sem deficiência hídrica), produtividade real (com deficiência hídrica), impacto da deficiência hídrica na produtividade (IRSH) e padrões de deficiência hídrica (sem deficiência hídrica - L, deficiência hídrica mediana - M, deficiência hídrica severa na fase reprodutiva - SR, deficiência hídrica terminal - T, deficiência hídrica na fase reprodutiva e terminal - RT) e seus respectivos desvios padrões (dp) para as TPEs milho (safra normal e safrinha).

\begin{tabular}{|c|c|c|c|c|c|c|c|c|c|}
\hline \multirow{3}{*}{ Solos } & \multirow{3}{*}{ Variável } & \multicolumn{4}{|c|}{ Milho (safra normal) } & \multicolumn{4}{|c|}{ Milho (safrinha) } \\
\hline & & \multicolumn{3}{|c|}{$\begin{array}{c}\text { Padrão de deficiência } \\
\text { hídrica }\end{array}$} & \multicolumn{5}{|c|}{$\begin{array}{c}\text { Padrão de } \\
\text { deficiência hídrica }\end{array}$} \\
\hline & & $\mathrm{L}$ & M & SR & Média & $\mathrm{L}$ & $\mathrm{T}$ & $\mathrm{RT}$ & Média \\
\hline \multirow[b]{3}{*}{$* \mathrm{CAD}$} & Produtividade potencial $\left(\mathrm{kg}, \mathrm{ha}^{-1}\right)$ & 7725 & 8117 & 8978 & 7908 & 8065 & 8342 & 8923 & 8317 \\
\hline & $\mathrm{dp}\left({\left.\mathrm{kg}, \mathrm{ha}^{-1}\right)}^{-}\right.$ & 1246 & 1211 & 1001 & & 799 & 980 & 1205 & \\
\hline & Produtividade real $\left(\mathrm{kg}, \mathrm{ha}^{-1}\right)$ & 7638 & 6161 & 1739 & 6900 & 7141 & 3639 & 786 & 3850 \\
\hline \multirow{3}{*}{$100 \mathrm{~mm}$} & $\mathrm{dp}\left(\mathrm{kg}, \mathrm{ha}^{-1}\right)$ & 1219 & 1797 & 1642 & & 1199 & 1582 & 906 & \\
\hline & IRSH $(\%)$ & 1 & 24 & 81 & 12 & 11 & 56 & 91 & 52 \\
\hline & Freqüência da deficiência hídrica (\%) & 62 & 34 & 4 & & 25 & 52 & 22 & \\
\hline \multirow[b]{3}{*}{ CAD } & Produtividade potencial $\left(\mathrm{kg}, \mathrm{ha}^{-1}\right)$ & 7430 & 7964 & 8480 & 7904 & 7983 & 8244 & 8781 & 8398 \\
\hline & $\mathrm{dp}\left(\mathrm{kg}, \mathrm{ha}^{-1}\right)$ & 1272 & 1238 & 1023 & & 966 & 918 & 1133 & \\
\hline & Produtividade real $\left(\mathrm{kg}, \mathrm{ha}^{-1}\right)$ & 7271 & 5661 & 945 & 5559 & 5547 & 2630 & 463 & 2214 \\
\hline \multirow{3}{*}{$50 \mathrm{~mm}$} & $\mathrm{dp}\left(\mathrm{kg}, \mathrm{ha}^{-1}\right)$ & 1218 & 1754 & 781 & & 1634 & 1574 & 575 & \\
\hline & IRSH $(\%)$ & 2 & 29 & 89 & 29 & 31 & 68 & 95 & 73 \\
\hline & Freqüência da deficiência hídrica (\%) & 20 & 71 & 9 & & 11 & 55 & 34 & \\
\hline
\end{tabular}

*Capacidade de água disponível. 

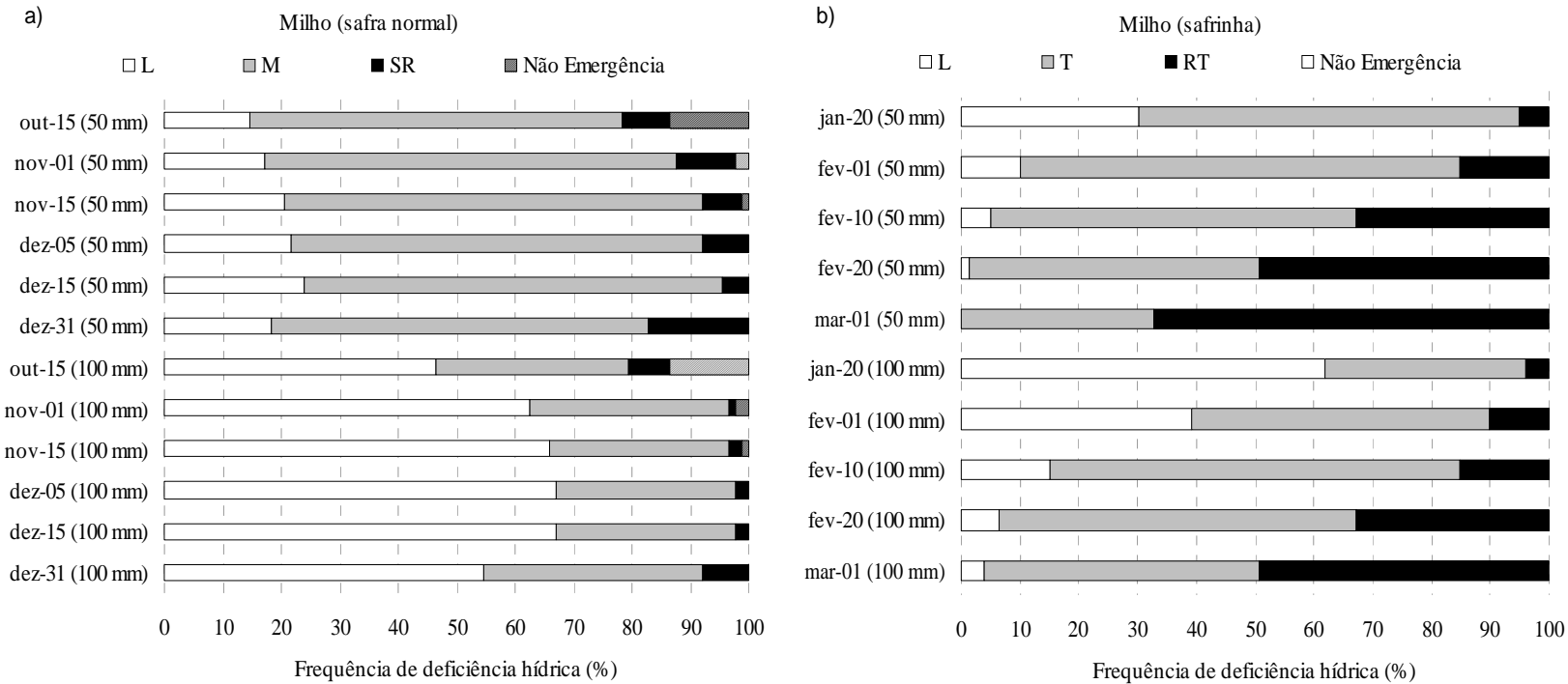

Figura 2 - Freqüência dos diferentes padrões de deficiência hídrica em função das datas de semeadura e cenários de solos com CAD $100 \mathrm{~mm}$ e $50 \mathrm{~mm}$, para a cultura de milho: safra normal (a) e safrinha (b), sendo (L) sem deficiência hídrica, (M) deficiência hídrica mediana, (SR) deficiência hídrica severa no período reprodutivo, (T) deficiência hídrica terminal e (RT) deficiência hídrica no período reprodutivo e terminal.

Para o milho safrinha, não houve ocorrência de falha na emergência em razão da falta de umidade no solo. Conforme ilustra a Figura $2 b$, a frequência da deficiência hídrica, para o milho safrinha, está diretamente relacionada com a data de semeadura. A partir da data de semeadura de 10/02 há um aumento na probabilidade de ocorrência dos padrões de deficiência hídrica T e RT, que são os mais prejudiciais à cultura.

\section{Impacto da deficiência hídrica na produtividade de grãos}

De acordo com Ceccarelli \& Grando (1991), em experimento realizado com cevada, reduções no rendimento de grãos maiores que $50 \%$, geralmente sob deficiência hídrica severa, a correlação positiva esperada entre a produtividade sob condições com e sem estresse por deficiência hídrica não é significativa. Edmeads et al. (2004) também reportaram que a correlação do rendimento de grãos entre híbridos de milho obtidos sob condições com e sem estresse por deficiência hídrica diminui em função da intensidade da deficiência hídrica aplicada. Isso pode ser decorrente do fato de que, nessas condições (deficiência hídrica severa), o genótipo não consegue expressar sua produtividade potencial, indicando a necessidade da avaliação direta da produtividade sob estresse por deficiência hídrica, nos programas de melhoramento de plantas.
Os resultados das simulações para a cultura do milho na safra normal indicaram que o IRSH médio para a TPE do milho safra normal foi menor que $50 \%$ para ambos os cenários, solos com CAD de $100 \mathrm{~mm}$ e $50 \mathrm{~mm}$ (Tabela 2). Os tipos de deficiência hídrica L e M representaram mais que $90 \%$ dos casos, em ambos os cenários. Assim, baseado nessa consideração, a deficiência hídrica não é a principal limitação para o desenvolvimento do milho no estado de Goiás, no período da safra normal.

Os padrões de deficiência hídrica obtidos para o milho na safrinha diferiram do milho safra normal. $\mathrm{O}$ desempenho do milho na safrinha é altamente dependente da data de semeadura. Para o milho na safrinha observaramse os maiores valores do IRSH médio (52\% para o solo com CAD de $100 \mathrm{~mm}$ e $73 \%$ para o solo com CAD de $50 \mathrm{~mm}$ ). De acordo com Fukai \& Cooper (2001), para condições de deficiência hídrica severa, como no caso do milho safrinha, há a necessidade de adotar procedimentos de escape da seca ou genótipos com mecanismos de tolerância à seca durante o enchimento de grãos. Atualmente, o procedimento utilizado em Goiás é o escape. Agricultores estão utilizando híbridos superprecoces, que exigem, para o seu desenvolvimento menos que 830 graus dia (Gadioli et al., 2000). Decorrente do fato de o processo de seleção e avaliação de genótipos de milho para a safrinha serem efetuados na época da safra normal (Tojo-Soler et al., 2001), 
condição que apresenta a menor probabilidade de ocorrência da deficiência hídrica no período de enchimento de grãos, reduz-se a probabilidade de se encontrar no mercado brasileiro genótipos de milho que possuam mecanismos que minimizem o efeito da seca na fase de enchimento de grãos.

\section{CONCLUSÕES}

A metodologia utilizada neste estudo pode ser aplicada para a determinação de padrões de estresses em um determinado ambiente-alvo. Entretanto, o sucesso dessa metodologia é altamente dependente da disponibilidade e qualidade da base de dados meteorológicos.

Para culturas de milho em safra normal, o estresse por deficiência hídrica não é o principal impedimento ao seu desenvolvimento. Entretanto, regiões que apresentam impedimento ao desenvolvimento do sistema radicular devem ter especial atenção para os períodos de semeadura visando a diminuir a probabilidade de ocorrência de deficiência hídrica severa.

Para a cultura de milho em safrinha, deve-se optar por variedades cultivadas ou híbridos superprecoces ou implantar programas de melhoramento para o desenvolvimento de variedades que minimizem o efeito da seca na fase de enchimento de grãos.

\section{REFERÊNCIAS BIBLIOGRÁFICAS}

AFFHOLDER, F. Empirically modeling the interaction between intensification and climatic risk in semiarid regions. Field Crops Research, Amsterdam, v.52, p.7993, 1997.

ALLEN, R.G.; PEREIRA, L.S.; RAES, D.; SMITH, M. Crop evapotranspiration-guidelines for computing crop water requirements. Rome: FAO, 1998. (FAO. Irrigation and drainage paper, 56).

BALBINO, L.C.; BRUAND, A.; BROSSARD, M.; GUIMARÃES, M. de F. Comportement de la phase argileuse lors de la dessiccation dans des Ferralsols microagrégés du Brésil: rôle de la microstructure et de la matière organique. Comptes Rendus de L' Academie des Sciences. Serie I: Sciences de La Terre et des Planètes, Montrouge, n.332, p.673-680, 2001.

BARON, C.; CLOPES, A.; PEREZ, P.; MULLER, B.; MARAUX, F. Manuels d'utilisation de SARRAZON. Montpellier: CIRAD, 1996. 29p.
CECCARELLI, S.; GRANDO, S. Environment of selection and type of germplasm in barley breeding for lowyielding conditions. Euphytica, Wageningen, v.57, p.207219, 1991.

CHAPMAN, S.; COOPER, M.; HAMMER, G.L.; BUTLER, D.G. Genotype by environment interactions affecting grain sorghum: II., frequencies of different seasonal patterns of drought stress are related to location effects on hybrid yields. Australian Journal Agricultural Science, Sydney, v.51, p.209-221, 2000a.

CHAPMAN, S.; HAMMER, G.L.; BUTLER, D.G.; COOPER, M. Genotype by environment interactions affecting grain sorghum: III., temporal sequences and spatial patterns in the target population of environments. Australian Journal Agricultural Science, Sydney, v.51, p.223-233, 2000b.

COOPER, M.; FOX, P.N. Environmental characterization based on probe and reference genotypes. In: COOPER, M.; HAMMER, G.L. (Eds.). Plant adaptation and crop improvement. Wallingford: CABI, 1996. p.529-547.

COOPER, M.; STUCKER, R.E.; DELACY, I.H.; HARCH, B.D. Wheat breeding nurseries, target environments, and indirect selection for grain yield. Crop Science, Madison, v.37, p.1168-1176, 1997.

EDMEADS, G.O.; BANZIGER, M.; SCHUSSLER, J.R.; CAMPOS, H. Improving abiotic stress tolerance in maize: a random or planned process? In:

INTERNATIONAL SYMPOSIUM ON PLANT

BREEDING, 2003, Mexico. Abstracts... Iowa: Iowa State University, 2004. p.397.

\section{EMPRESA BRASILEIRA DE PESQUISA}

AGROPECUÁRIA. Centro Nacional de Pesquisa de Solos. Sistema brasileiro de classificação de solos. Brasília, DF: Embrapa Produção da Informação; Rio de Janeiro: Embrapa Solos, 1999. 412p.

FUKAI, S.; COOPER, M. Development of drought resistant cultivars for rainfed lowland rice experience from Northeast Thailand and surrounding areas. In: INTERNATIONAL CONFERENCE ON THE IMPACT OF AGRICULTURAL RESEARCH FOR DEVELOPMENT IN SOUTHEAST ASIA, 2000 , Phnom Penh, Cambodia. Proceedings... Phnom Penh, 2001. p.185-194. 
GADIOLI, J.L.; DOURADO NETO, D.; GARCIA, Y.; GARCIA, A.; BASANTA, M.V. Air temperature, maize yield and phenological characterization associated to heat units. Scientia Agricola, Piracicaba, v.57, n.3, p.377383, 2000.

\section{INSTITUTO BRASILEIRO DE GEOGRAFIA E} ESTATÍSTICA. Banco de dados agregados. 2007.

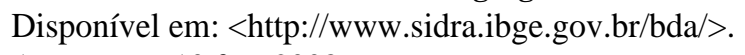
Acesso em: 10 fev. 2008.

KOURESSY, M.; DINGHUN, M.; VAKSMAN, M.; HEINEMANN, A.B. Adaptation to diverse semi-arid environments of sorghum genotypes having different plant type and sensitivity to photoperiod. Agricultural and Forest Meteorology, Amsterdam, v.148, p.357-371, 2008.

NIMER, E. Climatologia do Brasil. Rio de Janeiro: IBGE, 1989. 421p.

R DEVELOPMENT CORE TEAM. R: a language and environment for statistical computing. Vienna: $R$ Foundation for Statistical Computing, 2005. Disponível em: 〈http://[wWw_R-project.org $\rangle$. Acesso em: 15 fev. 2008.

SCHAFFERT, R.E.; ALVES, V.M.C.; PARENTONI, S.N.; RAGHTHAMA, K.G. Genetic control of phosphorus uptake and utilization efficiency in maize and sorghum under marginal soil conditions. In: RIBAUT, J.M.; POLAND, D. (Eds.). Molecular approaches for the genetic improvement of cereals for stable production in water-limited environments: a strategic planning workshop held at CIMMYT. Mexico: CIMMYT, 2000.

SINCLAIR, T.R.; LUDLOW, M.M. Influence if soil water supply on the plant water balance of four tropical grain legumes. Australian Journal of Plant Physiology, Victoria, v.13, p.329-341, 1986.

SULTAN, B.; BARON, C.; DINGKUHN, M.; JANICOT, S. Agricultural impacts of large-scale variability of the West African monsoon.

Agricultural and Forest Meteorology, Amsterdam, v.128, p.93-110, 2005.

TOJO-SOLER, C.; FOLEGATTI, M.V.; FARIA, R.T. Identification of the best genetic traits and agronomic practices for cornsowed out of season using the CERESMaize model. Revista Brasileira de Agrometeorologia, Santa Maria, v.9, n.2, p.339-346, 2002.

WARD, J.H. Hierarchical grouping to optimize an objective function. Journal of the American Statistical Association, Washington, v.58, p.236-244, 1963. 